\title{
Palm vein recognition scheme based on an adaptive Gabor filter
}

\author{
Prof.Sagar $\mathbf{B}^{1}$, Chaitra C $_{1}{ }^{2}$, Chaitra $\mathbf{J}^{3}$, Sampreetha $\mathbf{B} \mathbf{K}^{4}$,Vasumathi M G $\mathbf{G}^{5}$ \\ ${ }^{1}$ Professor EWIT, India, sagar11105@gmail.com \\ ${ }^{2}$ Student EWIT, India, chaithraputti2@ gmail.com \\ ${ }^{3}$ Student EWIT, India,chaitrajayanna1993cj@ gmail.com \\ ${ }^{4}$ Student EWIT, India, sammugowda@ gmail.com \\ ${ }^{5}$ Student EWIT, India,vasumathimg14@gmail.com
}

\begin{abstract}
Multibiometrics can provide higher identification accuracy than single biometrics, so it is more suitable for some real-world personal identification applications that need high-standard security. Among various biometrics technologies, palmprint identification has received much attention because of its good performance. We propose palm vein recognition scheme based on an adaptive 2D Gabor filter. Three key steps are studied in this scheme: region of interest (ROI) extraction, adaptive Gabor filtering, and template matching. First, in the palm vein image extraction step, the authors used the index finger on both sides of the valley to locate the square area, and then iteratively expanded the area of the square box to maximise the ROI. Second, in the feature extraction step, a parameter selection scheme was proposed for optimising the Gabor filter. Third, in the template matching step, the author presented a novel template matching algorithm referred to as the minimum normalised Hamming distance. Experimental results demonstrated that the scheme achieved good performance with an EER of $0.12 \%$.
\end{abstract}

Keywords: Biometrics, multibiometrics, palm vein recongnition.

\section{INTRODUCTION}

One of the important part of security access systems is Biometric identification techniques. Biometric information is harder to observe and can provide a higher security level against identity theft compared to traditional personal identification methods, such as personal identification numbers, magnetic swipe cards, keys, and smart cards. Among various biometric techniques, palm vein recognition is becoming more popular because it provides invisible internal biometric information and can be measured with a low-cost device. In this paper, we propose a palm vein recognition scheme. This scheme first located the valley on both sides of the index finger in the original image, determines the region of interest (ROI) area of the square, and then iteratively expanded the rectangular ROI to include as much biometric information as possible. The normalized image is then divided into several non-overlapping subregions and the optimal parameters of the Gabor filter were determined respectively from each sub-region. The extracted palm vein feature is then encoded into Vein Code format. In template matching the minimum normalized Hamming distance algorithm is applied, which returns the minimum distance value after multiple template displacements. This minimum distance value was used as the final score for template matching.

\section{RELATED WORK}

Acquiring palm veins procedure has been described in the literature. Investigated palm dorsal images are acquired using termal infrared device. Subcutaneous blood vessels absorb less radiation than their surroundings in the near-infrared spectrum of 780-1100 nm, resulting in high image contrast. Additionally, investigated hand vein image extraction under near infrared illumination. These studies have been fully described in vein image acquisition literature, but image recognition results obtained using different wavelengths are lacking. Palm vein recognition techniques are divided into four categories. First, geometrybased methods utilise line, curve, and point data to approximate the palm vein. Since these methods require coordinates for precise pattern comparison, the ROI extraction must calibrate position in advance. Second, statistics based methods use statistical information to recognise pattern features. These can be further divided into global and local static sub-categories. The local static subcategory includes local binary patterns (LBP), local derivative patterns (LDP), Weber local descriptors (WLD), and their variants, and are sensitive to scaling, rotation, and displacement. Previous studies applied Gabor filters to exploit the hand dorsal veins in a spatial domain with good results. On the other hand, the global static sub-category consists of invariant moments, wavelet moments, and gradient fields, and are invariant to scaling, rotation, and displacement. Thirdly, local invariant-based methods, such as the scale invariant feature transform (SIFT) and RootSIFT, and square root of SIFT (RootSIFT), extract local invariant palm vein features. However, since features extracted using these methods lack greyscale shift and corners, feature points are small and exhibit many intra-class changes. Fourth, appearance-based methods [11, 12], including principle component analysis (PCA), linear discriminant analysis (LDA), independent component analysis (ICA), and manifold methods acquire palm vein subspace coefficients as features without prior knowledge. This summary of prior work suggests that there is a lack of systematic comparison of different palm vein information processing steps, especially in feature extraction and matching. 
Sagar B et al., International Journal of Computing, Communications and Networking, 7(2) April - June 2018, 78-83

This has motivated us to further explore palm vein recognition in a more systematic way and to ascertain the best palm vein information processing approach.

\section{ARCHITECTURE}

The proposed palm vein recognition scheme consists of four basic modules, as shown in Fig. 1. Each of these modules plays a critical role in determining the palm vein matching performance in a biometric recognition system. The functionality of these modules is exhibited as follows:

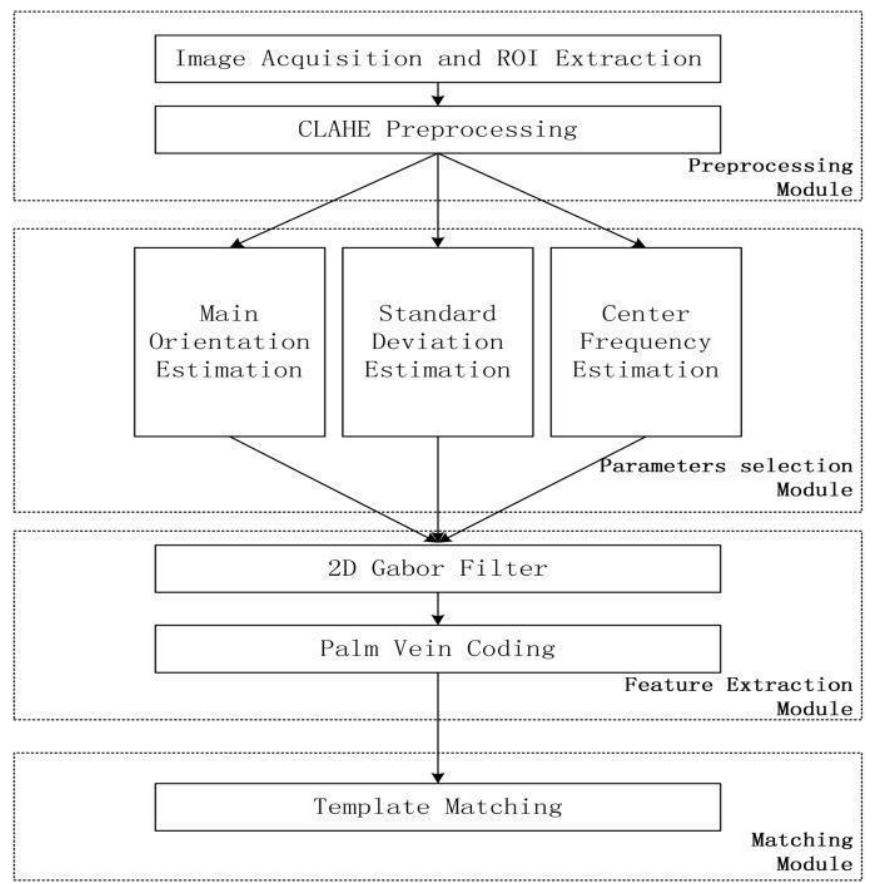

Fig. 1 Framework of our proposed palm vein recognition system.

i. Pre-processing module: it determines the ROI position for palm vein images acquired with an NIR illumination device.

ii. Parameter selection module: first, it divides the palm vein image into $8 \times 8$ non-overlapping sub regions, and estimates the parameters of a 2D Gabor filter containing main orientation, centre frequency, and standard deviation.

iii. Feature extraction module: it codes the palm vein feature into VeinCode format, in which the real and imaginary parts of the 2D Gabor result are stored in a binary feature matrix.

iv. Matching module: it compares the template with the target pattern using the minimum normalised Hamming distance method, which compensates for ROI calibration errors in the pre-processing step.

\section{IMAGE ACQUISITION AND ENHANCEMENT}

\subsection{Hand shape segmentation and ROI extraction}

Segmentation is an important step in separating the palm vein area from the original image. We used the OTSU method to extract hand contours from original greyscale images. The OTSU method, named after Nobuyuki Otsu, is an effective and automatic image thresholding method used for segmentation. To locate the correct position of an ROI, we located key points on the palm that contained peaks and valley points. Five red points and four green points represent finger tips and finger valleys, respectively. We defined the tip points $P$ t1 through $P$ t5 as the fingertips of the thumb, index, middle, ring, and little fingers, respectively. The valley points between those fingers were defined as $P \mathrm{v} 1$ through $P$ v4.

Several ROI location studies have used $P \mathrm{v} 2$ and $P \mathrm{v} 3$ to correctly locate the ROI, but the ROI size was not a maximum. Thus, to enlarge the ROI and accommodate more biometric information, we propose a novel ROI extraction method that can acquire square ROIs as large as necessary.

$d$ denotes the distance between valley point $P \vee 1$ and $P \mathrm{v} 2$, and $\theta$ denotes the angle between line segment $P \mathrm{v} 1 P \mathrm{v} 2$ and the vertical, respectively.

$$
\begin{aligned}
& d=(x P \mathrm{v} 1-x P \mathrm{v} 2) 2+(y P \mathrm{v} 1-y P \mathrm{v} 2) 2(1) \\
& \theta=\tan -1(x P \mathrm{v} 1-x P \mathrm{v} 2) \\
& (y P \mathrm{v} 1-y P \mathrm{v} 2) .(2)
\end{aligned}
$$

The bilinear grey value differential method was adopted to perform a rotation of $\theta$ degrees to ensure the line segment $P \mathrm{v} 1 P \mathrm{v} 2$ was horizontal. Then, the line segment $P \mathrm{v} 1 P \mathrm{v} 2$ was set as an edge of a square ROI box, and $P \mathrm{v} 2$ was fixed while the ROI was enlarged to the desired size. All elements in the ROI (square matrix) were 1 , and therefore we could scale up the square matrix dimensions iteratively until it no longer accommodated additional $1 \mathrm{~s}$. This method also provided maximising ROI size while preventing incorrect palm location. Fig. 2 shows the process of the proposed ROI method.

\subsection{Image enhancement}

Compared to the image taken in a white light environment, the contrast of the palm vein image captured by the NIR illumination capture device is relatively low. Therefore, direct extraction and feature matching will affect the performance of the biometric recognition system. Inspired by earlier studies using the CLAHE method for enhancing low-contrast images, this method increases the dynamic range of the image so that palm vein patterns can be more easily detected during feature extraction. 
Sagar B et al., International Journal of Computing, Communications and Networking, 7(2) April - June 2018, 78-83

As shown in Fig. 3, this method improved contrast between the vein pattern and the background and limited surrounding noise. For convenience in the feature extraction step, we normalized the ROI size into $256 \times 256$ pixels using a bilinear interpolation scale method.

\section{FEATURE EXTRACTION}

\subsection{Overview of 2D adaptive Gabor filter}

In the computer vision, the feature analysis function of the gabor filter is very powerfull because the simple cell of the mammalian cortical are to be used in the profile field. Such as irises, facrs, palam print and faces are researched in previous process of gabor filter to be exploit in different biometric field. An oriented complex process of the circular 2D gabor filter by the modulated $2 \mathrm{D}$ process of Gaussian functions expressed as $\mathrm{G} \sigma, \mu, \theta \mathrm{x}, \mathrm{y}$ $=g \sigma x, y \cdot \exp 2 \pi j \mu x \cos \theta+y \sin \theta$ where $j=-1$ and $g \sigma x, y$ is a Gaussian envelope defined as $g \sigma x, y=12 \pi \sigma 2 \cdot \exp -(\mathrm{x} 2+\mathrm{y} 2) 2 \sigma 2$.

In this process, the standard deviation of a Gaussian envelop

are denoted by $\sigma$. The frequency of the span-limited sinusoidal grating denoted by $\mu$.

The orientation in the interval denoted by $\theta$, range between

0०-180。. Using Euler's formula, the $\mathrm{G} \sigma, \mu, \theta \mathrm{x}, \mathrm{y}$ term can be denoted into a real part $\mathrm{R} \sigma, \mu, \theta \mathrm{x}, \mathrm{y}$.

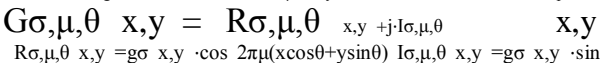

$\mathrm{R} \sigma, \mu, \theta \mathrm{x}, \mathrm{y}=\mathrm{g} \sigma \mathrm{x}, \mathrm{y}$
$2 \pi \mu(\mathrm{x} \cos \theta+\mathrm{y} \sin \theta)$

The slight of a gabor filter of a uniform luminance is known as direct current(DC).

\subsection{Estimation of main orientation}

For every palm vein sub region in the ROI, the feature of the continuous line-like texture tendency is socalled principle direction. It is the intrinsic property of the palm vein and also it represents the orientation of the parameter $\theta$ in the $2 \mathrm{D}$ gabor filter process. We can adopt a randam transform-based method rather than $3 \times 3$ pixels direction process.

$\theta=\operatorname{argmax} \varphi \sum \mathrm{x} 0=1 \mathrm{n} \sum \mathrm{y} 0=1 \mathrm{mDk} x 0, \mathrm{y} 0=\varphi$, where $\varphi \in 0 \circ, 30 \circ, 60 \circ, 90 \circ, 120 \circ, 150$ 。

\subsection{Estimation of standard deviation}

The representation of standard deviation of a Gaussian distribution are denoted by $\sigma$. The gabor filter of a $2 \mathrm{D}$ process will be related to the width of the modulation. The standard deviation will be measured in the local region variance of the image $D(I(i, j))$. The image texture will be fixed value according to the process it will change the images.

$$
\begin{aligned}
& E(I(i, j))=\sum i=1 m \sum j=1 n I(i, j) m \times n, \\
& D(I(i, j))=\sum i=1 m \sum j=1 n I((i, j))-E(I(i, j)) 2 m \times n,
\end{aligned}
$$

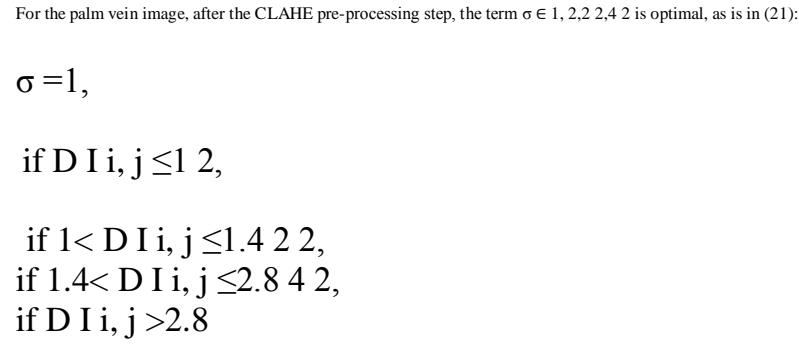

These values indicate a stable area, a slowly changing area, a moderately changing area.

\subsection{Estimation of center frequency}

The main direction of the changing grey level are seen as a sine wave. The calculation of the distance between 2edges or 2 ridges can be calculated. The center frequency $\mu=1 / T$. In the palm vein recognition system, the method will not be sufficient due to the fact of the pattern is too be blurry between the edges and ridges.

$$
\begin{aligned}
& \mu=0, \text { if } \sigma=1 \\
& \mu=0.12, \text { if } \sigma= \\
& 2 \mu=0.8, \text { if } \sigma \\
& =22 \mu=2, \text { if } \sigma \\
& =42
\end{aligned}
$$

\subsection{Palm vein feature coding}

According to the above method, we we can independently estimated the $\sigma, \mu$, and $\theta$. parameter for a $2 \mathrm{D}$ gabor filter for each sub region. The size of the neighborhood window size will be $S \times S$, with $S=2 K+1$, the image of the real part,

$\mathrm{CR} \sigma, \mu, \theta(\mathrm{x}, \mathrm{y})$ and the images of the 2D gabor filter are $\mathrm{CI \sigma}, \mu, \theta(\mathrm{x}, \mathrm{y})$, the whole image of ROI was traversed. CR $\sigma, \mu, \theta(\mathrm{x}, \mathrm{y})=\sum \mathrm{i}=-\mathrm{k} \mathrm{k} \sum \mathrm{j}=-\mathrm{k} \mathrm{k}(\mathrm{I}(\mathrm{x}+\mathrm{i}), \mathrm{y}+\mathrm{j} \cdot \mathrm{R} \sigma, \mu, \theta(\mathrm{x}, \mathrm{y})$

Finally, features extracted from the palm vein image were encoded into a binary form VR,VI , i.e. the VeinCode, as defined in (25) and (26), respectively:

$$
\begin{aligned}
& \operatorname{VR}(\mathrm{x}, \mathrm{y})=1 \text {, if } \mathrm{CR} \sigma, \mu, \theta \mathrm{x}, \mathrm{y} \geq 0 \\
& 0 \text {, if } \mathrm{CR} \sigma, \mu, \theta \mathrm{x}, \mathrm{y}<0 \text {, } \\
& \mathrm{VI}(\mathrm{x}, \mathrm{y})=1 \text {, if CI } \sigma, \mu, \theta \mathrm{x}, \mathrm{y} \geq 0 \\
& 0 \text {, if } \mathrm{CI} \sigma, \mu, \theta \mathrm{x}, \mathrm{y}<0
\end{aligned}
$$


Sagar B et al., International Journal of Computing, Communications and Networking, 7(2) April - June 2018, 78-83

\section{TEMPLATE MATCHING}

\subsection{Displacement compensation}

Since ROI calibration, it will lead some degree of displacement deviation in the palm print image. Finally it will affect the performace of a recognition system, by keeping the template palm vein image fixed and moving the target palm view image aong with vertical direction and horizontal directions.

The ROI images were $256 \times 256$ pixels, the possible region was $256 \times 448$ pixels

\subsection{Minimum normalised Hamming distance}

Based on Lee's normalised Hamming distance (NHD) method, we propose the minimum normalised Hamming distance (MNHD) algorithm, the binary vein code matrix of the palm vein pattern features are encoded. PR and QR represent the real parts after Gabor convolution; PI and QI represent the imaginary parts after Gabor convolution; $\otimes$ denotes a Boolean XOR operator; the size of the feature matrix is $\mathrm{N} \times \mathrm{N} ; \mathrm{S}$ and $\mathrm{T}$ denote the maximum allowable offset in the horizontal and vertical directions, respectively, and $\mathrm{Hx}=\min \mathrm{N}, \mathrm{N}+\mathrm{x}-\max 1,1+\mathrm{x}$.

$\operatorname{DDM}=\operatorname{NHD}(1,1) \mathrm{NHD} 1,2 \mathrm{NHD} 2,1 \mathrm{NHD}(2, \mathrm{n}) \cdots \operatorname{NHD}(1, \beta) \cdots \operatorname{NHD}(2, \beta) !$

$$
\text { NHD 9 } \alpha, \beta) \text {. }
$$

In our palm vein recognition system, the threshold of maximum displacement compensation was 8 pixels, and thus the displacement distance matrix was $8 \times 8$.

\section{EXPERIMENTAL RESULTS}

\subsection{Database}

CASIA-MS-palm printVI database was conducted by the

evaluation experiments [19] (CASIA database). Using a noncontact device, 7200 palm images are in CASIA database. 100 different peoples are captured all images, and each sample contains six palm images at different wavelengths of light (460, 630, 700, 850, and 940nm).

We selected the $940 \mathrm{~nm}$ wavelength group. Furthermore, to increase the number of inter-class sample comparisons, we regarded the left hand and right hand samples as being from different people.

\subsection{Performance evaluation of proposed method}

The power matching experiments was conducted in between genuine and imposter groups. The intra- and inter- class matches that are included by the genuine and imposter groups. The matching between a samplew and other samples in the same class is defined by the Intra-class matching, whereas the matching between a sample and other samples belonging to a different class is defined by the inter class matching. To ecaluate our proposed method we adopted the minimum normalized hamming distance.

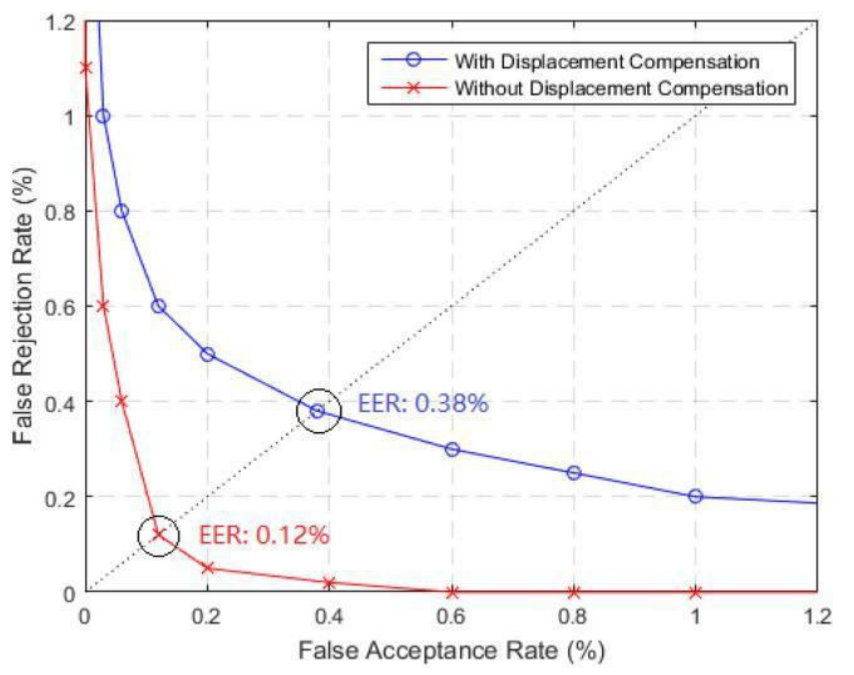

We evaluated the equal error rate (EER) of our proposed method by making trade-offs between the false rejection rate (FRR) and the false acceptance rate (FAR). This is shown in the receiver operating characteristic curve (ROC) where the EER of our proposed method was $0.12 \%$. To confirm the robustness of the displacement compensation scheme in the matching step, we evaluated recognition performance using the proposed palm vein recognition method based on two types of matching schemes: with displacement compensation and without displacement compensation.

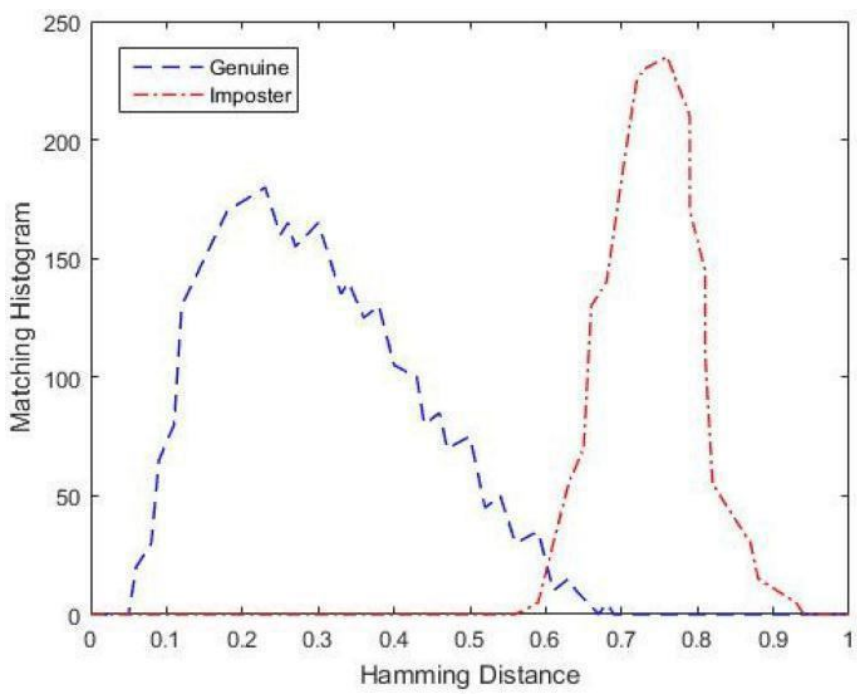

Fig. 1 Hamming distance measurement histogram 
Sagar B et al., International Journal of Computing, Communications and Networking, 7(2) April - June 2018, 78-83

\subsection{COMPARISON AND DISCUSSION}

To further demonstrate the effectiveness of the proposed method, To existing conventional methods with conducted detailed comparisons. We wrote codes based on the corresponding publications, To run the algorithms of these conventional method. EER of our proposed method was $0.12 \%$ is illustrates in Table 2 which is slightly better than other methods.

The local statistical information such as minutiae points and minutiae feature and hessian phase method extract locations and ridge boforcations for palm vein texture. However, when texture is unclear or indistinguishable, these methods fail to extract geometric feature information. In contrast, the Laplacian palm method extracts features using LPP and aims to preserve the local structure of the image space, while the eigenvein method extracts features using (2D)2 PCA and aims to preserve the global structure of the image space. The performance of both the Laplacian palm and eigenvein methods was lower than our method.

\subsection{Computational efficiency} algorithm.

We analysed the time complexity of the proposed For the core code of the feature extraction steps, the estimation of main orientation' function must perform an operation in each direction, and therefore the time complexity is $O n$. Finally, we conducted experiments to compare time-consumption of different methods. These experiments were conducted using MATLAB 8.6 on a Windows 64-bit system with an Intel i5-4690 CPU with 8 GB RAM. Our proposed method was more competitive by comprehensive comparison, as shown in. Experimental results showed that our proposed method required $1.28 \mathrm{~s}$ of computation time for the feature extraction step, and was the most efficient of all methods. the feature extraction step, and was the most efficient of all methods.
Algorithm: MNHD matching

$$
\begin{aligned}
& \text { 01. /Input } \\
& \text { 02. F target Itarget feature matrix } \\
& \text { 03. F } F_{\text {template }} \text { Itemplate feature matrix } \\
& \text { 04. Ddeviation /ldisplacement deviation pixels } \\
& \text { 05. HOutput } \\
& \text { 06. R Result } \quad / 1 \text { for accept, } 0 \text { for reject } \\
& \text { 07. /Initialize } \\
& \text { 08. matrix } D D M \text { / thas }\left(2 D_{\text {deviation }}+1\right)^{2} \text { dimensions } \\
& \text { 09. /Begin algorithm } \\
& \text { 10. FOR } \alpha=-D_{\text {deviation }} T 0 D_{\text {deviation }} \text { ? } \\
& \text { 11. FOR } \beta=-D_{\text {deviation }} \text { TO } D_{\text {deviation }} \\
& \text { 12. Get the recognition area from overlapped region } \\
& \text { between target and template feature matrix. } \\
& \text { 13. Calculate the NHD using Eq. (27). } \\
& \text { 14. Save current } \operatorname{NHD}(\alpha, \beta) \text { value to matrix DDM. } \\
& \text { 15. }\} \\
& \text { 16. }\} \\
& \text { 17. IF Min }\{\text { all elements in DDM }\} \leq T_{\text {threshold }} \\
& \text { 18. RETURN } R_{\text {Result }}=1 \text { /laccept } \\
& \text { 19. ELSE } \\
& \text { 20. RETURN } R_{\text {Result }}=0 \text { /reject } \\
& \text { 21. /End algorithm }
\end{aligned}
$$

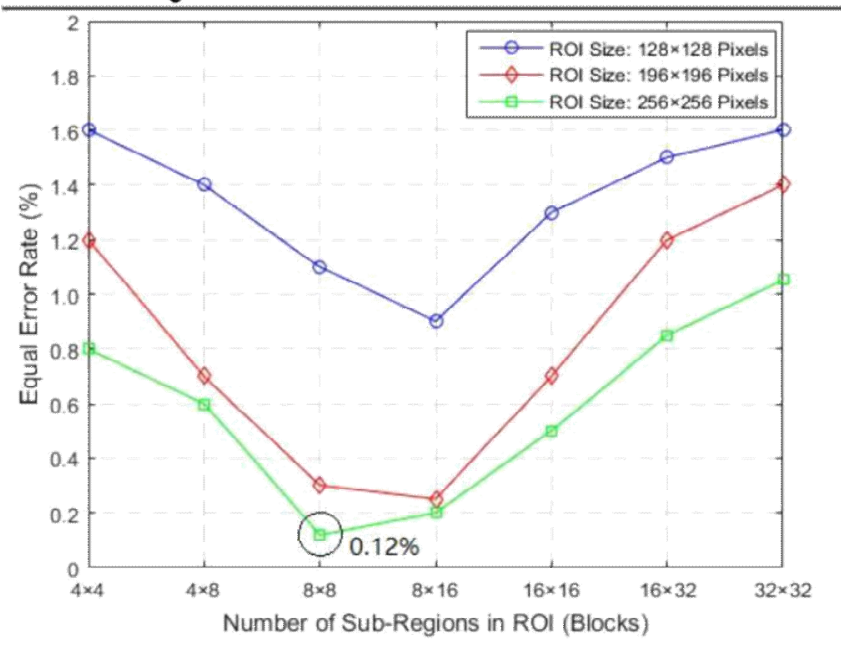

Fig. 3 Illustrations of EER curve for different ROI and segmentation methods 
Sagar B et al., International Journal of Computing, Communications and Networking, 7(2) April - June 2018, 78-83

\section{CONCLUSION}

To acquire square ROIs designed the simple and efficient ROI location method was proposed, to obtain maximal biometric information. In this paper we also presenting a Novel adaptive Gabor filter parameter estimation approach. The innovative aspects of this approach are as follows: To determine the local Gabor direction parameter we used the Random method, determine the local Gabor variance parameter for degree of image change, and Determine its central frequency parameter for inswnsitively of the local Gabor filter. Experimental results shows that our scheme achieved good performance with an EER of $0.12 \%$. compaire to other conventional palm vein recognition method. This performance was superior.

\section{ACKNOWLEDGEMENT}

We sincerely thank for providing the CASIA-MS-Palmprint VI database for Chinese Academy of Sciences' Institute of Automation for providing the used in this research.

\section{REFERENCES}

[1] Lee, J.C., Lee, C.H., Hsu, C.B., et al.: 'Dorsal hand vein recognition based on 2D Gabor filters', Imaging Sci. J., 2014, 62, (3), pp. 127-138

https://doi.org/10.1179/1743131X12Y.0000000049

[2] Lin, C.L., Fan, K.C.: 'Biometric verification using thermal images of palmdorsa vein patterns', IEEE Trans. Circuits Syst. Video Technol., 2004, 14, (2), pp. 199-213

https://doi.org/10.1109/TCSVT.2003.821975

[3] Cross, J.M., Smith, C.L.: 'Thermographic imaging of the subcutaneous vascular network of the back of the hand for biometric identification'. Int. Carnahan Conf. on Security

Technology, Sanderstead, UK, October 1995, pp. 20-35

https://doi.org/10.1109/CCST.1995.524729

[4] Zhou, Y., Kumar, A.: 'Human identification using palmvein images', IEEE Trans. Inf. Forensics Sec., 2011, 6, (4), pp. 1259-1274

https://doi.org/10.1109/TIFS.2011.2158423

[5] Song, W., Kim, T., Kim, H.C., et al.: 'A finger-vein verification system using mean curvature', Pattern Recognit. Lett., 2011, 32, (11), pp. 1541-1547

https://doi.org/10.1016/j.patrec.2011.04.021

[6] Mirmohamadsadeghi, L., Drygajlo, A.: 'Palm vein recognition with local binary patterns and local derivative patterns'. Int. Joint Conf. on Biometrics, Washington, USA, October 2011, pp. 1-6

https://doi.org/10.1109/IJCB.2011.6117804

[7] Chen, J., Shan, S., He, C., et al.: 'WLD: a robust local image descriptor', IEEE Trans. Pattern Anal. Mach. Intell., 2009, 32, (9), pp. 1705-1720

https://doi.org/10.1109/TPAMI.2009.155

[8] Lee, J.-C.: 'A novel biometric system based on palm vein

image', Pattern Recognit. Lett., 2012, 33, (12), pp. 1520-1528

https://doi.org/10.1016/j.patrec.2012.04.007

[9] Li, X., Guo, S., Gao, F., et al.: 'Vein pattern recognitions by moment invariants'. Int. Conf. on Bioinformatics \& Biomedical Engineering, Wuhan, China, July 2007, pp. 612615

https://doi.org/10.1109/ICBBE.2007.160

[10] Kang, W.X., Liu, Y., Wu, Q.X., et al.: 'Contact-free palm-vein recognition based on local invariant features', PLoS One, 2014, 9, (5), pp. 1239-1245

https://doi.org/10.1371/journal.pone.0097548

[11] Liu, Z., Yin, Y., Wang, H., et al.: 'Finger vein recognition with manifold learning', J. Netw. Comput. Appl., 2010, 33, (3), pp. 275-282

https://doi.org/10.1016/j.jnca.2009.12.006

[12] Yang, G., Xi, X., Yin, Y.: 'Finger vein recognition based on (2D)(2) PCA and metric learning', J. Biomed. Biotechnol., 2012, 2012, (3), p. 324249

[13] Kang, W.X., Wu, Q.X.: 'Contactless palm vein recognition using a mutual foreground-based local binary pattern', IEEE Trans. Inf. Forensics Sec., 2014, 9, (11), pp. 1974-1985

https://doi.org/10.1109/TIFS.2014.2361020

[14] Mehrotra, R., Namuduri, K.R., Ranganathan, N.: 'Gabor filter-based edge detection', Pattern Recogn., 1992, 25, (12), pp. 1479-1494

https://doi.org/10.1016/0031-3203(92)90121-X

[15] Han, W.Y., Lee, J.C.: 'Palm vein recognition using adaptive Gabor filter', Expert Syst. Appl., 2012, 39, (18), pp. 13225-13234

https://doi.org/10.1016/j.eswa.2012.05.079

[16] Jia, W., Huang, D.-S., Zhang, D.: 'Palmprint verification based on robust line orientation code', Pattern Recogn., 2008, 41, (5), pp. 1504- 1513

https://doi.org/10.1016/j.patcog.2007.10.011

[17] Zhou, Y.J., Liu, Y.Q., Feng, Q.J., et al.: 'Palm-vein classification based on principal orientation features', PLoS One, 2014, 9, (11), p. 12

https://doi.org/10.1371/journal.pone.0112429

[18] Yue, F., Zuo, W., Zhang, D., et al.: 'Orientation selection using modified FCM for competitive code-based palmprint recognition', Pattern Recogn., 2009, 42, (11), pp. 2841-2849

https://doi.org/10.1016/j.patcog.2009.03.015

[19] 'CASIA-MS-PalmprintV1',http://biometrics.idealtest.org/, accessed 20 January 2016

[20] Wang, J.-G., Yau, W.-Y., Suwandy, A., et al.: 'Fusion of palmprint and palm vein images for person recognition based on 'Laplacianpalm' feature'. 2007 IEEE Conf. on Computer Vision and Pattern Recognition, Minneapolis, USA, June 2007, pp. 1-8 